\title{
MLC-1 (MYOSIN LIGHT CHAINS-1) IN DIFFERENTIAL DIAGNOSIS OF DYSPNEA
}

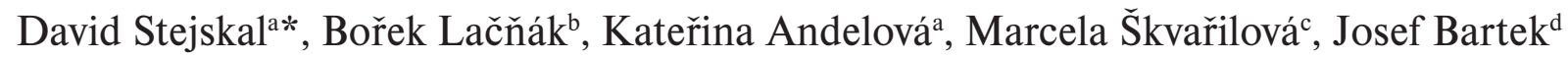 \\ a Department of Laboratory Medicine, Hospital Šternberk, Czech Republic \\ ${ }^{b}$ Department of Internal Medicine, Hospital Šternberk, Czech Republic \\ $2^{\text {nd }}$ Clinic of Internal Medicine, Faculty Hospital Charles University Prague, Czech Republic \\ ${ }^{d}$ Institute of Medical Chemistry and Biochemistry, Faculty of Medicine, Palacký University Olomouc, Czech Republic \\ e-mail:david.stejskal@quick.cz
}

Received: April 7, 2005; Accepted (with revisions): June 3, 2005

Key words: MLC-1/NT-proBNP/Dyspnea/Heart failure

Myosin light chains-1 (MLC-1) have been recently associated with the markers of heart function (NYHA, LVEF, NT-proBNP).

Verification of the relationship between markers of heart function (New York Heart Association classification (NYHA), left ventricle ejection fraction determination (LVEF), $\mathrm{N}$ terminal prohormone of natriuretic peptide $\mathrm{B}$ type BNP (NT-proBNP) and concentrations of myosin light chains-1 (MLC-1) was assesed.

Patients examined for dyspnea without signs of acute coronary syndrome. All patients underwent echocardiography (calculation of left ventricle ejection fraction - LVEF) and in the serum of all subjects NT-proBNP (ELEIA) and MLC-1 (ELISA) were determined.

In the 38 patients ( 21 men, 17 women), mean age of 58 years ( \pm 12 years as $1 \mathrm{SD})$, a significant negative correlation was found between NT-proBNP and LVEF $(r=-0.47 ; p=0.02$, Spearman). The median levels of NT pro-BNP were closely associated with NYHA classification (type II - $584 \mathrm{ng} / \mathrm{l}$, type III - $2792 \mathrm{ng} / 1$, type IV - $6400 \mathrm{ng} / \mathrm{l} ; \mathrm{p}<$ 0.05). Individuals with clinical NYHA IV differed significantly in median MLC-1 concentrations from persons with clinical NYHA classification II and III (type II - $5.7 \mathrm{ng} / 1$, type III - $8.9 \mathrm{ng} / 1$, type IV - $17 \mathrm{ng} / \mathrm{l} ; \mathrm{p}<0.05$ ). A significant negative correlation between MLC-1 and LVEF $(-0.35 ; \mathrm{p}<0.03)$ and significant positive correlations between MLC-1 and NT-proBNP (- 0.42; p <0.012) were found. In conclusion MLC-1 cannot be used as a diagnostic marker in differential diagnosis of dyspnea.

\section{INTRODUCTION}

Recently, several papers have reported on the necessity of new sensitive and particularly early tests of myocardial ischemia and function, which would allow an early and efficient therapeutic intervention. A promising laboratory marker may therefore be MLC-1 (myosin light chain -1). Only a few reports describe good diagnostic validity of this marker; MLC-1 appears to have a high diagnostic validity for the assessment of minimal myocardial damages.

Several recent papers also have reported the relationship between concentrations of myosin light chains- 1 (MLC-1) and myocardial function; individuals with heart failure had significantly higher levels of MLC- $1^{1-5}$. There is no valid information about MLC-1 assessment in diagnosing myocardial dyspnea.

The aim of the present study was use of sera MLC-1 to differentiate cardiogenic from non-cardiogenic dyspnea.

\section{METHODS}

The study comprised patients at the Department of Internal Medicine, Hospital Sternberk, who were examined for dyspnea but who had no signs of acute coronary syndrome. All subjects were diagnosed on the basis of defined criteria - anamnesis, clinical examination, electrocardiography, X-ray examination of heart and lungs with cardio-thoracal index calculation, spirometry, lung scintigraphy, laboratory examination and response to a given therapy ${ }^{6}$. All underwent echocardiographic examination (with calculation of left ventricle ejection fraction - LVEF - using Simpson method).

Blood samples was drawn under aseptic precautions from vena cubiti, after several minutes rest in half-sitting position, the serum sample was separated in a cooled centrifuge at $4{ }^{\circ} \mathrm{C}$ with $3000 \mathrm{~g}$ and subsequently frozen at $-80^{\circ} \mathrm{C}$. All persons were examined for serum MLC-I concentration (MLC-I, sandwich ELISA, Biovendor, Brno, The Czech Republic) with satisfying analytical characteristics (intrassay coefficient of variation $(\mathrm{CV})=7.4 \%$, interassay $\mathrm{CV}=8.3 \%), \mathrm{N}$ terminal prohormone of natriuretic peptide type B (NT-proBNP, Elecsys 2010, Roche ELEIA analyser, Basil, Switzerland) was determined with satisfactory analytical characteristics, too. Concentrations of NT-proBNP and MLC-1 were determined after defrosting in the course of one day.

The study was approved by the ethical commission of the Hospital Šternberk. 
The data obtained were processed by means of the software Medcalc (Medcalc, Mariakerke, Belgium). The value $\mathrm{p}<0.05$ was considered as statistically significant.

\section{RESULTS}

The group of 38 patients ( 21 men and 17 women), mean age of 58 years ( $1 \mathrm{SD}= \pm 12$ years) was examined (Table 1).

It was found that the value of NT-proBNP showed a linear negative correlation with left ventricle ejection fraction $(r=-0.47, p=0.02$, Spearman $)$.

The median values of NT pro-BNP were closely associated with NYHA classification (type II - $584 \mathrm{ng} /$; type III - 2792 ng/l; type IV - 6400 ng/l; p < 0.05, KruskalWallis).

Only individuals with clinically severe heart failure (NYHA IV) differed significantly in median MLC-1 concentration from persons with clinically less severe signs of heart failure (NYHA II-III); type II - $5.7 \mathrm{ng} / 1$, type III - 8.9 ng/1, type IV - 17 ng/1 ( $\mathrm{p}>0.05$, Kruskal-Wallis) (Table 2).

Significant negative correlations were found between MLC-1 and LVEF ( $\mathrm{r}=-0.35, \mathrm{p}=0.03$, Spearman); positive correlations between MLC-1 and NT-proBNP ( $r=0.42 ; p=0.01$, Spearman) were recorded.

Division of the group under study according to the cause of dyspnea (cardiogenic versus non-cardiogenic dyspnea) revealed only a significant difference in NT-proBNP
( $\mathrm{p}<0.01$, Kruskal-Wallis); increased MLC-1 concentrations in patients with cardiogenic dyspnea were not statistically significant ( $p>0.05$, Kruskal-Wallis) (Table 3 ).

The cut-off of MLC-1 >1.9 mg/1 (optimized with receive operating curve - ROC) showed $75 \%$ MLC-1 sensitivity and $61 \%$ specificity for cardiogenic dyspnea. Given an insufficient area below the ROC curve (AUC $=0.67$; $95 \% \mathrm{CI}=0.50-0.82)$ it is concluded that this parameter cannot be used for differential diagnosis of dyspnea (in contrast to NT-proBNP: $90 \%$ sensitivity and $70 \%$ specificity, $\mathrm{AUC}=0.81)($ Fig. 1).

\section{DISCUSSION}

The present study showed a close correlation between NYHA classification, left ventricle ejection fraction and NT-proBNP values was found.

Myosin is a contractile protein consisting of two heavy chains (MW 200 kD) and two pairs of light chains. Pairs of light chains are called MLC-1 (MW $25 \mathrm{kD}$ ) and MLC-2 (MW $19 \mathrm{kD}$ ). In the organism there are several types of MLC (skeletal muscles MLC - sMLC, ventricle MLC - vMLC, aorta MLC - aMLC, etc.). Impaired integrity of cardiomyocytes (during myocardial necrosis) leads to a release of vMLC-1 from the myocardium into circulation; increased MLC-1 occurs even 3-6 hours after the accident and maximal levels are found during $1^{\text {st }}$ to $5^{\text {th }}$ day after necrosis (heavy chains occur in the circulation during $2^{\text {nd }}$ to $10^{\text {th }}$ day after necrosis, maximal values are

Table 1. NT - proBNP and MLC -1 values in observed group.

\begin{tabular}{|c|c|c|c|c|c|}
\hline Parameter & Unit & Mean & SD & Median & Normality \\
\hline NT-proBNP & $\mathrm{ng} / 1$ & 2084 & 4798 & 4021 & No \\
\hline MLC-1 & $\mathrm{ng} / 1$ & $7 . \mathrm{III}$ & 11.0 & $1 . \mathrm{III}$ & No \\
\hline
\end{tabular}

Normality assessed by Komolgorov-Smirnov test

Table 2. NT- proBNP and MLC -1, stratified according to NYHA classification.

\begin{tabular}{|c|c|c|r|r|c|}
\hline Parameter & Unit & \multicolumn{1}{|c|}{ NYHA II } & NYHA III & NYHA IV & $\mathrm{p}$ \\
\hline NT-proBNP & $\mathrm{ng} / 1$ & 584 & 2792 & 6400 & $<0.05$ \\
\hline MLC-1 & $\mathrm{ng} / 1$ & 5.700 & 8.900 & 17 & $<0.05^{*}$ \\
\hline
\end{tabular}

* Only differences between NYHA IV and NYHA III or NYHA

Table 3. NT-proBNP and MLC-1 in patients with cardiogenic/non-cardiogenic dyspnea.

\begin{tabular}{|c|c|c|c|c|c|c|c|}
\hline Parameter & \multicolumn{2}{|c|}{ Cardiogenic dyspnea } & & \multicolumn{3}{|c|}{ Non-cardiogenic dyspnea } & Difference \\
\hline \multirow{2}{*}{ NT-proBNP } & Mean & SD & Median & Mean & SD & Median & \\
\cline { 2 - 8 } & 4569 & 7608 & 1527 & 224 & 549 & 111,8 & $\mathrm{p}<0.00001$ \\
\hline MLC-1 & 10.200 & 13.80 & 4.700 & 4.300 & 6.200 & 1.350 & NS \\
\hline
\end{tabular}

NS $-p>0.05$ 


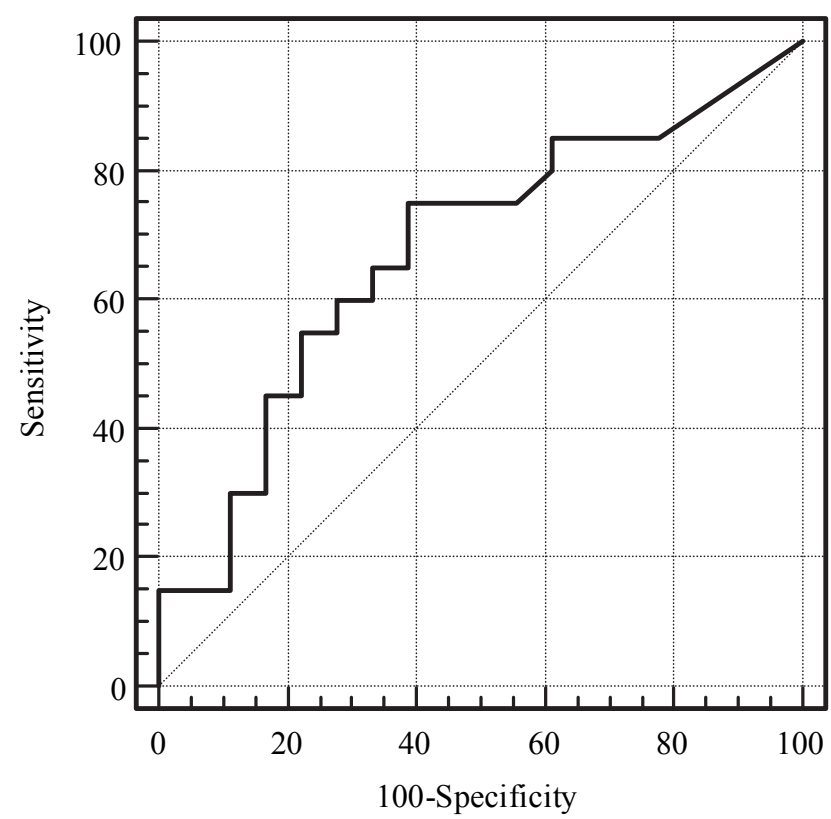

Fig. 1. ROC: MLC-1 senzitivity and specifity for cardiogenic dyspnea.

recorded during $5^{\text {th }}$ to $6^{\text {th }}$ day). Increased MLC in the circulation lasts for one week. As MLC is excreted by the kidney, persons with impaired kidney function showing higher MLC levels and dynamics of changes are impaired after myocardial necrosis.

To date, several papers have reported on increased MLC-1 in stabilized patients with myocardial dysfunction (NYHA III); MLC-1 concentration may be used for mortality prediction in these patients ${ }^{1,5}$. One reason for increased MLC-1 levels may be degeneration of myofibrils and release of their components into circulation, often found in myocardial dysfunction. Moreover, these structural changes may accelerate dysfunction of ventricles and enhance the filling pressure ${ }^{6,8-10}$. Another reason may be MLC-1 gene overexpression. This factor may lead to an oversynthesis of protein in myocardial cells and its transfer into circulation. However, it remains unclear if such transfer is associated with an impaired structure of cardiomyocytes (if impaired structure is prerequisite).

Enhanced mortality followed up in patients with increased MLC-1 may reflect myocytic stress, impaired integrity of myocytic membrane, or impaired structure of cardiomyocytes that are accompanied with release of intracellular proteins into circulation. Interestingly enough is a report on this phenomenon found in the clinically stabilized patients ${ }^{7}$.
The present study confirmed the hypothesis of an association between NYHA classification, left ventricle ejection fraction (LVEF) and NT-proBNP concentration and MLC-1 level in dyspneic patients (various types of dyspnea)

It is concluded that the results here clearly indicate that the use of MLC-1 as a marker for the differential diagnosis of dyspnea is unsuitable. This may be due to various regulatory mechanisms affecting the final concentration of MLC-1 in venous blood (in contrast to NT-proBNP). Our findings confirm recent reports on increased MLC-1 in clinically stabilized patients with normal or slightly increased NT-proBNP concentration (this may be due to the myocytic stress).

\section{REFERENCES}

1. Hansen MS, Stanton EB, Gawad Y. (2002) Relation of Circulationg Cardiac Myosin Light Chain 1 Isoform in Stable Severe Congestive Heart Failure to Survival and Treatment With Flosequinan. Am Journal of Cardiology 90, 969-973.

2. Hillis GS, Zhao N, Dalsey WC. (1999) Utility of cardiac troponin I, creatine kinase-MB mass, myosin light chain 1, and myoglobin in the early in-hospital triage of „high risk“ patients with chest pain. Heart 82, 614-320.

3. Sawada H, Nagata I, Shoraki K. (2003) Serum Concentration of MLC-1 and Left ventricular Shortening Fraction of Neonates. Yonago Acta Medica 42, 69-78.

4. Goto T, Takase H, Tariyama T. (2003) Circulating concentrations of cardiac proteins indicate the severity of congestive heart failure. Heart 89, 1303-1307.

5. Communal C, Sumandea M, Tombe P. (2002) Functional consequenses of caspase activation in cardiac myocytes. PNAS 99, 6252-6256.

6. Špinar J, Vítovec K. (1992) Radiologic Changes in chronic heart failure. Cor et Vasa 34, 88-99.

7. Nageh T, Chin D, Cooke JC, Meehan A, Monaghan MJ, Sherwood RA. (2002) Interpretation of plasma brain natriuretic peptide concentrations may require adjustment for patient's age. Ann Clin Biochem 39, 151-153.

8. Ishii J, Nomura M, Nakamura Y, Naruse H, Mori Y, Ishikawa T, Ando T, Kurokawa H, Kondo T, Nagamura Y, Ezaki K, Hishida H. (2002) Risk stratification using a combination of cardiac troponin $\mathrm{T}$ and brain natriuretic peptide in patients hospitalized for worsening chronic heart failure. Am J Cardiol 89, 691-695.

9. Lubien E, DeMaria A, Krishnaswamy P, Clopton P, Koon J, Kazanegra R, Gardetto N, Wanner E, Maisel AS. (2002) Utility of B-natriuretic peptide in detecting diastolic dysfunction: comparison with Doppler velocity recordings. Circulation 105, 595-596.

10. Harrison A, Morrison LK, Krishnaswamy P, Kazanegra R, Clopton P, Dao Q, Hlavin P, Maisel AS. (2002) B-type natriuretic peptide predicts future cardiac events in patients presenting to the emergency department with dyspnea. Emerg Med 39, 131-138. 